\begin{tabular}{|c|l|}
\hline Title & Relationship of forces acting on implant rods and degree of scoliosis correction \\
\hline Author(s) & Salmingo, Remel A lingal an; Tadano, Shigeru; Fuji isaki, Kazuhiro; A be, Y uichiro; Ito, Manabu \\
\hline Citation & $\begin{array}{l}\text { Clinical biomechanics, 28(2), 122-128 } \\
\text { https://doi.org/10.1016/.Clinbiomech.2012.12.001 }\end{array}$ \\
\hline Issue Date & 2013.02 \\
\hline Doc URL & http://hdl.handle.net/2115/52754 \\
\hline Type & article (author version) \\
\hline File Information & HUSCA PO40113.pdf \\
\hline
\end{tabular}

Instructions for use 


\section{RELATIONSHIP OF FORCES ACTING ON IMPLANT RODS AND DEGREE OF SCOLIOSIS CORRECTION}

Remel Salmingo, MEng ${ }^{1 *}$, Shigeru Tadano, $\mathrm{PhD}^{1 *}$, Kazuhiro Fujisaki, $\mathrm{PhD}^{1 *}$, Yuichiro Abe, $\mathrm{MD}, \mathrm{PhD}^{2} *$, Manabu Ito, $\mathrm{MD}, \mathrm{PhD}^{3 *}$

1 Laboratory of Biomechanical Design, Division of Human Mechanical Systems and Design, Graduate School of Engineering, Hokkaido University, North 13 West 8 Kita-ku, Sapporo, Japan

2 Department of Orthopaedic Surgery, Eniwa Hospital, Kogane-Cho 2-1-1, Eniwa, Japan

3 Advanced Medicine for Spine and Spinal Cord Disorders, Graduate School of Medicine, Hokkaido University, North 15 West 7 Kita-ku, Sapporo, Japan

\section{Corresponding Author:}

Shigeru Tadano, $\mathrm{PhD}$

tel: +81117066405

fax: +81117066405

e-mail: tadano@eng.hokudai.ac.jp

Total number of words excluding abstract and references: 2654

Number of words of the abstract: 249

Number of tables: $\mathbf{3}$

Number of figures: 8 
*Authors contributed equally to this work and no funding was received for this study. There is no conflict of interest to be reported.

\section{ABSTRACT}

Background: Adolescent idiopathic scoliosis is a complex spinal pathology characterized as a three-dimensional spine deformity combined with vertebral rotation. Various surgical techniques for correction of severe scoliotic deformity have evolved and became more advanced in applying the corrective forces. The objective of this study was to investigate the relationship between corrective forces acting on deformed rods and degree of scoliosis correction.

Methods: Implant rod geometries of six adolescent idiopathic scoliosis patients were measured before and after surgery. An elasto-plastic finite element model of the implant rod before surgery was reconstructed for each patient. An inverse method based on Finite Element Analysis was used to apply forces to the implant rod model such that it was deformed the same after surgery. Relationship between the magnitude of corrective forces and degree of correction expressed as change of Cobb angle was evaluated. The effects of screw configuration on the corrective forces were also investigated.

Findings: Corrective forces acting on rods and degree of correction were not correlated. Increase in number of implant screws tended to decrease the magnitude of corrective forces but did not provide higher degree of correction. Although greater correction was achieved with higher screw density, the forces increased at some level.

Interpretation: The biomechanics of scoliosis correction is not only dependent to the corrective forces acting on implant rods but also associated with various parameters such as screw placement configuration and spine stiffness. Considering the magnitude of forces, increasing screw density is not guaranteed as the safest surgical strategy. 
Force and degree of scoliosis correction

Keywords: Adolescent idiopathic scoliosis, screw density, rod deformation, corrective force, biomechanics, bone 


\section{INTRODUCTION}

Adolescent idiopathic scoliosis, a complex spinal disease, is characterized as a three-dimensional deformity of the spine combined with vertebral rotation. Various surgical techniques for correction of severe scoliotic deformity have evolved and became more advanced in applying the corrective forces threedimensionally. These techniques are the rod derotation technique by Cotrel and Dubousset (CD), Ventral Derotation Spondylodesis (VDS), Halm-Zielke Instrumentation (HZI), Simultaneous Double Rod Rotation Technique (SDRRT) and Direct Incremental Segmental Translation (DIST) (Cotrel et al., 1988; Halm et al., 1998; Ito et al., 2010; Wang et al., 2011; Zielke et al., 1982).

Decision-making on the levels of instrumentation/fixation, types and number of implants, shape and size of implant rod is still dependent on individual surgeon's experience (Desroches et al., 2007; Majdouline et al., 2009). Optimal scoliosis surgical treatment is not achieved due to the variability of surgeons' preferences and different correction objectives. Thus, simulation of surgeon's preferences and correction objectives using a computer model is important to understand the biomechanics of scoliosis correction. Several biomechanical models were developed to simulate various surgical steps and strategies. These were used to determine the advantages and disadvantages between different instrumentation systems by comparing the distribution of corrective forces acting at the vertebrae (Wang et al., 2011a; Wang et al., 2011b). Furthermore, preoperative surgical planning was made possible using patient-specific finite element models (Aubin et al., 2003; Aubin et al., 2008). These studies estimated the suitable surgical strategy for scoliosis operation, however, the results might be unrealistic because rod deformation was not considered in their analyses (Aubin et al., 2003; Aubin et al., 2008; Desroches et al., 2007; Lafon et al., 2009). The 
implant rod geometry was obtained only from postoperative data. Their reported magnitude of forces (in several hundred or even thousand Newtons) was apparently high that should have deformed the implant rod during the surgical treatment. Thus, the postoperative geometry of rod could not be used as a substitute for the initial implant rod geometry. Careful investigation of the geometrical changes of rod and corrective forces acting on it are also important to fully understand the scoliosis correction mechanism (Salmingo et al, 2012a; Salmingo et al., 2012b). Moreover, relationships between the magnitude of corrective forces, number of screws, screw placement configuration and degree of correction need to be further elucidated.

The objective of this study was to analyze the corrective forces acting on the deformed implant rod after the surgical treatment of scoliosis. The relationships between the magnitude of corrective forces, degree of correction and absolute number of screws were determined. We also considered the screw density defined as the percentage of number of pedicle screws used over the number of pedicle screws that could have been used within the implant rod length. The relationships between screw density, magnitude of corrective forces and degree of correction were also investigated.

\section{MATERIALS AND METHODS}

\subsection{Patients and implant rod deformation}

Six scoliosis patients were diagnosed as severe adolescent idiopathic scoliosis. All patients were surgically operated in the same orthopaedic department after completion of the requirements set by the ethics committee of the university hospital. Implant rods and polyaxial pedicle screws of USS II Polyaxial system 
(Synthes GmbH, Oberdorf, Switzerland) were used. The implant rod diameter was $6 \mathrm{~mm}$. The implant rod length varies with each scoliosis patient. All rods were prebent only at a single plane. Implant rods and screws were surgically implanted following the Simultaneous Double Rod Rotation Technique (SDRRT) procedure (Ito et al., 2010). In SDRRT, two rods were inserted into the polyaxial screw heads. The polyaxial screw heads remained untightened until the rod rotation was completed; allowing the rod to rotate and translate freely inside the screw head. A rod rotating device was used to hold and rotate the rod. A torque was applied to rotate the rod (approx. $90^{\circ}$ ) at the same time to create corrective forces on rods through the screws to deform the spine into normal shape. Figure 1(a) shows the radiograph of corrected spine after the surgical treatment of scoliosis. The initial geometry of rod was measured before surgical implantation, Fig. 1(b). The final geometry of rod was measured a week (maximum) after surgery by Aquilion 64 CT scanner (Toshiba Medical Systems Corp., Tochigi, Japan). In order to standardize the rod three-dimensional axes, the coordinate system proposed by the Scoliosis Research Society was used (Yeung et al, 2003). The positive $x$-axis, $y$ axis, and $z$-axis is directed toward the anterior, left lateral side and superior direction, respectively. Force analysis of rod at the convex side was neglected because the CT imaging could not significantly detect deformation of rod along that side in all patients. On the other hand, the rods at the concave side were significantly deformed after the surgical treatment of scoliosis. The changes of implant rod geometry at the concave side of each patient were used in this study. The images of implant rod at the concave side before and after surgery were imported into CAD software Solidworks 2010 (Dassault Systemes, Massachusetts, USA) to measure the geometry and deformation. 


\subsection{Finite Element Analysis}

Figure 2 shows the procedure for calculating the corrective forces using Finite Element Analysis (FEA) (Salmingo et al., 2012a). FEA was performed using ANSYS 11.0 software (ANSYS, Inc., Pennsylvania, USA). The elasto-plastic finite element model of the implant rod before surgery was reconstructed using 10 node tetrahedral solid elements. The forces were applied iteratively to the location of screws such that the rod was deformed the same after surgery. The corrective forces acting on the implant rod were obtained after series of iterations using the force optimization algorithm proposed by Salmingo et al. (2012a). Initially, zero force $F_{i}(i=$ no. of screws $)$ was applied to the corresponding location of each screw on the rod geometry before surgery, in Fig. 2 (upper right). The elastoplastic deformation analysis was performed. The displacement vector $\vec{e}_{i}$ required to attain the location of each screw after surgery was used in the iteration process. The applied forces were replaced by adding the value of the displacement vector $\vec{e}_{i}$. The whole process was repeated until the displacement vector $\vec{e}_{i}$ was minimized. The evaluating function defined as the sum of the squares of displacement vector $\overrightarrow{\vec{e}}_{i}$ expressed as

$$
\sum_{i=1}^{n}\left|\overrightarrow{\mathrm{s}}_{i}\right|^{2}<\omega
$$

was used to evaluate each iteration process. The force iteration process was stopped when the evaluating function was less than $\propto$ (where $\propto=0.5$ ). During 
this time, the rod model was deformed the same rod geometry after surgery because the displacement vector $\overrightarrow{\vec{e}}_{i}$ was already minimized.

The elasto-plastic material model was based from the implant manufacturer specifications. Material properties were elastic modulus $(E)$, yield stress $\left(\sigma_{Y}\right)$, yield strain $\left(\varepsilon_{Y}\right)$ and hardening coefficient $(H)$ equal to $105 \mathrm{GPa}, 900$ $\mathrm{MPa}, 8.57 \times 10^{-3}$ and $2.41 \mathrm{GPa}$, respectively.

\subsection{Degree of Correction}

Frontal radiographs were taken before and after surgery for Cobb angle measurements. The degree of correction $\Delta \theta$ was referred to as the difference between the preoperative Cobb angle $\theta_{1}$ and postoperative Cobb angle $\theta_{2}$ (Table 1). The degree of correction was also expressed as correction rate. This was computed as the ratio of the degree of correction over the preoperative Cobb angle $\Delta \theta / \theta_{1}$

\subsection{Screw Density}

The screw density was defined as the number of pedicle screws used divided by the number of pedicle screws that could have been used within the implant rod length. Screw density was expressed as percentage. The screw density at the convex side of the deformity was excluded since the rod at that side was not significantly deformed after the surgical treatment of scoliosis.

\subsection{Pullout and Push-in Force}

Since the calculated forces correspond also the pullout and push-in forces acting at the vertebrae of the scoliosis patient. The magnitudes of pullout and push-in forces were also estimated from the applied forces. The pullout or push-in force was defined as the pulling or pushing force acting parallel to the endplate of the vertebra at the sagittal plane, in Fig. 1(c). This was computed using the reaction 
force (i.e. acting at the spine in the opposite direction) of the computed corrective force that deformed the rod during scoliosis surgery. The rod geometry was used to define the direction of the pullout or push-in force since its curvature constitutes the spine curvature after the surgical treatment of scoliosis. The rod geometry was approximated by quintic polynomial function using the method proposed by Salmingo et al. (2012b). The tangent angle (broken line) that is orthogonal to the pullout or push-in force axis was computed by evaluating the first derivative of quintic polynomial function for location of each screw. The reaction force was resolved into component (Reaction force $\times \operatorname{Cos} \beta$ ), equal to the magnitude of pullout or push-in force acting at the corresponding vertebra level.

\section{RESULTS}

The magnitude of corrective forces obtained by FEA acting on each screw is listed in Table 2. The corrective force acting at the apical vertebra was also obtained. The results show that the corrective forces along the apical vertebra tended to increase. This indicates that the apical vertebra needs higher corrective forces than the other vertebra. For cases where the screw was not placed in the apical vertebra, the closest force acting at the apex was used (or whichever was higher in case that the closest force could not be determined; i.e. Patient 6). This supports the fact that vertebrae near the apex needed higher corrective force. The maximum forces were located at the extreme fixation levels. Most of them are at the lumbar side. The absolute number of screws, screw density and magnitude of corrective forces were summarized in Table 3. 


\subsection{Degree of correction}

Table 1 shows the correction rate of each scoliosis patient. The correction rate was mean 69\% (SD 11\%). Patient 6 and 5 gained the lowest and highest correction rate, respectively. An increase in absolute number of screws did not significantly correlate with the degree of correction $(r=0.48, p>0.05)$, Fig. 3. On the other hand, the screw density correlated well with degree of correction $(\mathrm{r}=0.81, \mathrm{p}<$ 0.025), in Fig. 4. Figure 5 shows the relationship between the average force and degree of correction. A significant correlation could not be found between the average force $(r=0.03, p>0.05)$ and the degree of correction. In total, the magnitude of forces did not correlate well with the degree of correction.

\subsection{Number of screws}

Figure 6 shows the relationships between the absolute number of screws and magnitude of forces. A decreasing trend was found between the number of screws and average force, however, the correlation was not significant $(r=0.63$ and, $p>$ 0.05), in Fig. 6(a). Conversely, there was a significant negative correlation between the force acting at/near the apical vertebra $(\mathrm{r}=0.73, \mathrm{p}<0.05)$ with increasing number of screws, Fig. 6(b).

\subsection{Screw density}

The screw density at the concave side of each patient was obtained. The summation of forces and maximum force of patients did not correlate well with increasing screw density $(r=0.04$ and $r=0.28, p>0.05$ respectively), in Figs. 7(a) and 8(b). Likewise, a trend could not be established for both relationships. For some patients, the summation of forces and maximum forces are relatively lower than patient who had $100 \%$ screw density. Thus, having higher implant density is not guaranteed as the safest surgical strategy if we consider the magnitude of forces. 


\subsection{Pullout and Push-in Force}

The pullout or push-in forces acting at the vertebrae of scoliosis patients were computed, in Fig. 8. The results show that almost all patients had push-in force at the most inferior and superior level, whereas the apical region had pullout forces. Furthemore, the values of pullout forces indicate that the magnitude is increasing at the apical region.

\section{DISCUSSION}

Numerical studies involving patient-specific finite element models have reported possible clinical outcomes of different surgical strategies through preoperative planning. However, these studies lack detailed modeling of implant rod because the initial rod geometry was not measured before surgery. Relationships of various parameters such as the magnitude of corrective forces, number of implants used, degree of correction and implant placement configuration are not yet well understood. Furthermore, it has already been shown by previous studies that too high corrective forces can cause implant breakage or bone fracture which may lead to pullout of screws from the vertebra (Patwardhan et al., 2010; Paxinos et al., 2012). Thus, analysis of corrective forces acting on deformed rod is important to understand the biomechanics of scoliosis correction.

\subsection{Degree of Correction}

The results in this study showed that the degree of correction does not depend on the absolute number of screws. Implant placement configuration such as screw density contributed to higher degree of correction. This suggests that vertebrae can be easily manipulated if more implants are attached nearer to each other. Indeed, Patient 6 (having lowest screw density) and Patient 5 (having the highest 
screw density) gained the lowest and highest correction rate, respectively. Although implant and screw density was considered differently, findings in this study are in agreement with the previous study of Clements et al. (2009). We defined screw density as the percentage of number of pedicle screws used over the number of pedicle screws that could have been used within the implant rod length, whereas they defined implant density as the ratio of implants used over the number of available implants sites within the measured Cobb angle. We believe that the implants sites within the implant rod length have significant role in deformity correction than the implants sites beyond the implant rod length. Screw density at the convex side was not also included because the implant rod was not deformed after surgery. The rod at the convex side might have limited role in deformity correction but provides additional mechanical stability after it has been fixed by the screws.

All in all, the magnitude of forces did not correlate well with the degree of correction. The results suggest that scoliosis correction mechanism is not only dependent to the corrective forces. Some parameters such as flexibility or stiffness of the scoliotic spine need to be considered. Intuitively, patients having stiffer scoliotic spines need higher corrective forces than patients who have more flexible scoliotic spines. The current magnitude of forces must have been affected by patient's flexibility or stiffness. Thus, scoliotic spine's flexibility or stiffness is one of the major factors that influence the outcome of the clinical operation. Little et al. (2012) showed that flexibility is a critical factor that governs the degree of deformity correction and there is still much to be learned about the complex relationships which determine the individual patient's flexibility. 


\subsection{Number of screws}

Although not statistically significant, a decreasing trend was found between the magnitude of forces and number of screws. The magnitude of corrective forces tends to be more distributed with increasing absolute number of screws. However, increase in number of screws increases the surgical time that could also result to increased blood loss (Rose et al., 2009). All the same, the previous relationship showed that the increase in number of screw did not result to higher degree of correction.

\subsection{Screw density}

The maximum and summation of forces indicate that having $100 \%$ screw density does not always guarantee safe clinical outcome. Some patients have much lower values of maximum and summation of forces than the patient who had $100 \%$ screw density. Further studies involving various screw placement configurations including distribution of uninstrumented levels could decrease the magnitude of forces while achieving similar correction outcome.

\section{CONCLUSION}

Scoliosis correction mechanism does not only depend on the number of screws and corrective forces acting on implants but also associated with screw placement configuration. Our findings suggest that scoliosis deformity can be easily manipulated with higher screw density. However, increasing screw density alone does not ensure safe clinical outcome as indicated by the magnitude of forces. In this study, the corrective forces analyzed from implant rod deformation reveal new insights on correction of scoliosis deformity. 


\section{REFERENCES}

Aubin, C., Labelle, H., Chevrefils, C., Desroches, G., Clin, J., Biovin, A., 2008. Preoperative planning simulator for spinal deformity surgeries. Spine 33, 2143-2152.

Aubin, C., Petit, Y., Stokes, I., Poulin, F., Gardner, M.M., Labelle, H., 2003. Biomechanical modeling of posterior instrumentation of the scoliotic spine. Comp. Methods in Biomech. and Biomed. Eng. 6, 27-32.

Clements, D., Betz, R., Newton, P., Rohmiller, M., Marks, M., Bastrom, T., 2009. Correlation of scoliosis curve correction with the number and type of fixation anchors. Spine 34, 2147-2150.

Cotrel, Y., Dubousset, J., Guillaumat, M., 1988. New universal instrumentation in spinal surgery. Clin. Orthop. 227, 10-23.

Desroches, G., Aubin, C.E., Sucato, D., Rivard, C.H., 2007. Simulation of an anterior spine instrumentation in adolescent idiopathic scoliosis using a flexible multi-body model. Med. Bio. Eng. Comput. 45, 759-768.

Halm, H.F., Liljenqvist, U., Niemeyer, T., Chan, D.P., Zielke, K., Winkelmann, W., 1998. Halm-Zielke instrumentation for primary stable anterior scoliosis surgery: Operative technique and 2-year results in ten consecutive adolescent idiopathic scoliosis patients within a prospective clinical trial. Eur. Spine J. 7, 429-434.

Ito, M., Abumi, K., Kotani, Y., Takahata, M., Sudo, H., Hojo, Y., Minami, A. 2010. Simultaneous double rod rotation technique in posterior instrumentation surgery for correction of adolescent idiopathic scoliosis. J. Neurosurg. Spine 12, 293-300. 
Lafon, Y., Lafage, V., Dubousset, J., Skalli, W., 2009. Intraoperative threedimensional correction during rod rotation technique. Spine 34:512-19.

Little, J.P., Izatt, M.T., Labrom, R.D., Askin, G.N., Adam, C.J., 2012. Investigating the change in three dimensional deformity for idiopathic scoliosis using axially loaded MRI. Clin. Biomech. 27, 415-421.

Majdouline, Y., Aubin, C.E., Sangole, A., Labelle, H., 2009. Computer simulation for the optimization of instrumentation strategies in adolescent idiopathic scoliosis. Med. Bio. Eng. Comput. 47, 1143-1154.

Mehta, H., Santos, E., Ledonio, C., Sembrano, J., Ellingson, A., Pare, P., Murrell, B., Nuckley, D., 2012. Biomechanical analysis of pedicle screw thread differential design in an osteoporotic cadaver mode. Clin. Biomech. 127, $234-240$.

Paxinos, O., Tsitsopoulos, P.P., Zindrick, M.R., Voronov, L.I., Lorenz, M.A., Havey, R.M., Patwardhan, A.G. 2012 Evaluation of pullout strength and failure mechanism of posterior instrumentation in normal and osteopenic thoracic vertebrae. J. Neurosurg. Spine. 13, 469-476.

Rose, P.S., Lenke, L.G., Bridwell, K.H., Mulconrey, D.S., Cronen, G.A., Buchowski, J.M., Schwend, R.M., Sides, B.A., 2009. Pedicle Screw Instrumentation for Adult Idiopathic Scoliosis: An Improvement Over Hook/Hybrid Fixation. Spine 34, 852-857.

Salmingo, R., Tadano, S., Fujisaki, K., Abe, Y., Ito, M., 2012. Corrective force analysis for scoliosis from implant rod deformation. Clin. Biomech. 27, 545-550.

Salmingo, R.A., Tadano, S., Fujisaki, K., Abe, Y., Ito, M., 2012. A Simple Method for In Vivo Measurement of Implant Rod Three-Dimensional Geometry During Scoliosis Surgery. ASME J. Biomech. Eng. 134, 054502. 
Wang, X., Aubin, C., Crandall, D., Labelle, H., 2011a. Biomechanical comparison of force levels in spinal instrumentation using monoaxial versus multi degree of freedom postloading pedicle screws. Spine 36, 95-104.

Wang, X., Aubin, C.E., Crandall, D., Labelle, H., 2011b. Biomechanical modeling and analysis of a direct incremental segmental translation system for the instrumentation of scoliotic deformities. Clin. Biomech. 26, 548-555.

Yeung, A., Ottaviano, D., Lee, D., Haher, T., 2003. Spine testing modalities, in: DeWald, R., Arlet, V., Carl, A., O'Brien, M., (Eds.), Spinal deformities the comprehensive text, Thieme Medical Publishers Inc., New York, pp. 176186.

Zielke, K., 1982. Ventral derotation spondylodesis: Results of treatment of cases of idiopathic Lumbar scoliosis. Z. Orthop. Ihre. Grenzgeb. 120, 320-329. 


\section{LIST OF TABLES}

Table 1 Clinical data of scoliosis patients.

Table 2 Magnitude of corrective forces acting at each vertebra level.

Table 3 Summary of number of screws, screw density and magnitude of forces of each patient.

\section{LIST OF FIGURES}

Figure 1 (a) Postoperative radiograph of operated scoliotic spine (b) Corrective force acting at the screw of deformed rod. (c) Pullout force computed from the reaction force of corrective force.

Figure 2 Procedure for corrective force analysis using FEA, redrawn (Salmingo et al., 2012a).

Figure 3 Relationship between the number of screws and degree of correction.

Figure 4 Relationship between screw density and degree of correction.

Figure 5 Relationship between degree of correction and average force.

Figure 6 Relationships between number of screws and magnitude of forces. (a) Number of screws vs. average force (b) Number of screws vs. force at/near apical vertebra

Figure 7 Relationships between screw density and magnitude of forces. (a) Screw density and summation of forces and (b) Screw density and maximum force Figure 8 Pullout and push-in forces acting at the vertebra of each patient. 


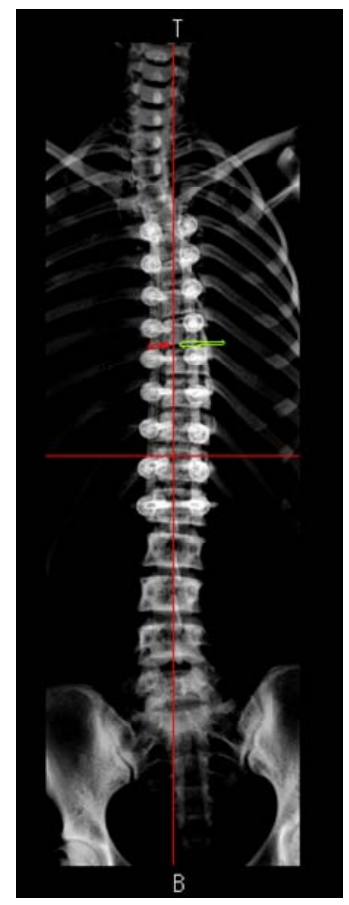

Frontal

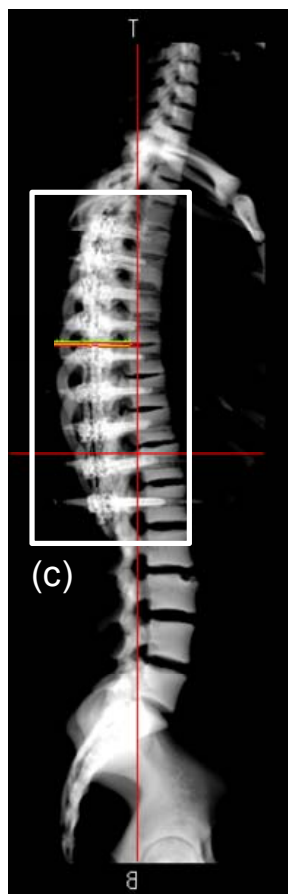

Sagittal

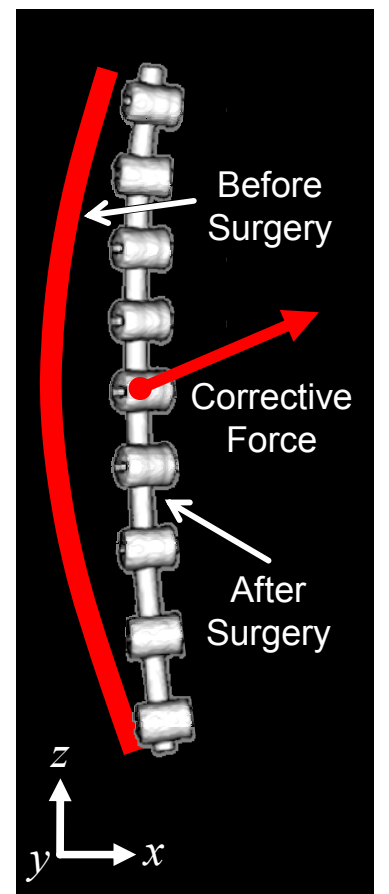

Rod deformation

(a)

(b)

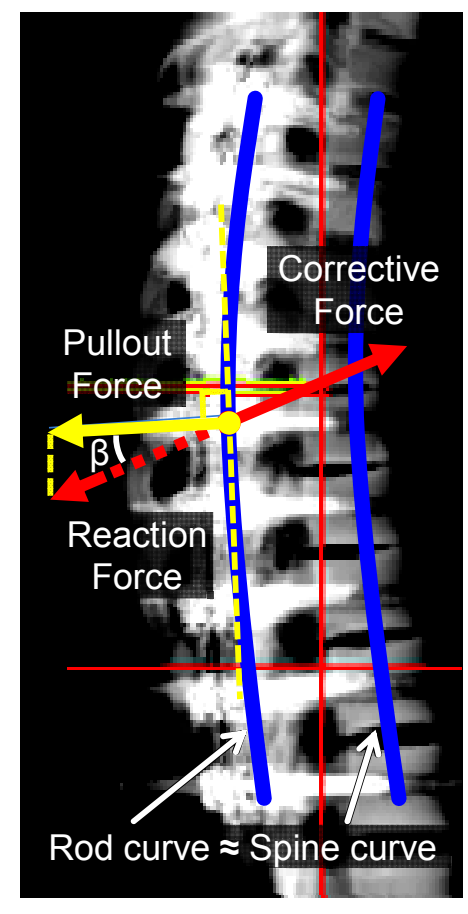

Vertebral pullout force

(c)

Figure 1 (a) Postoperative radiograph of operated scoliotic spine (b) Corrective force acting at the screw of deformed rod. (c) Pullout force computed from the reaction force of corrective force. 


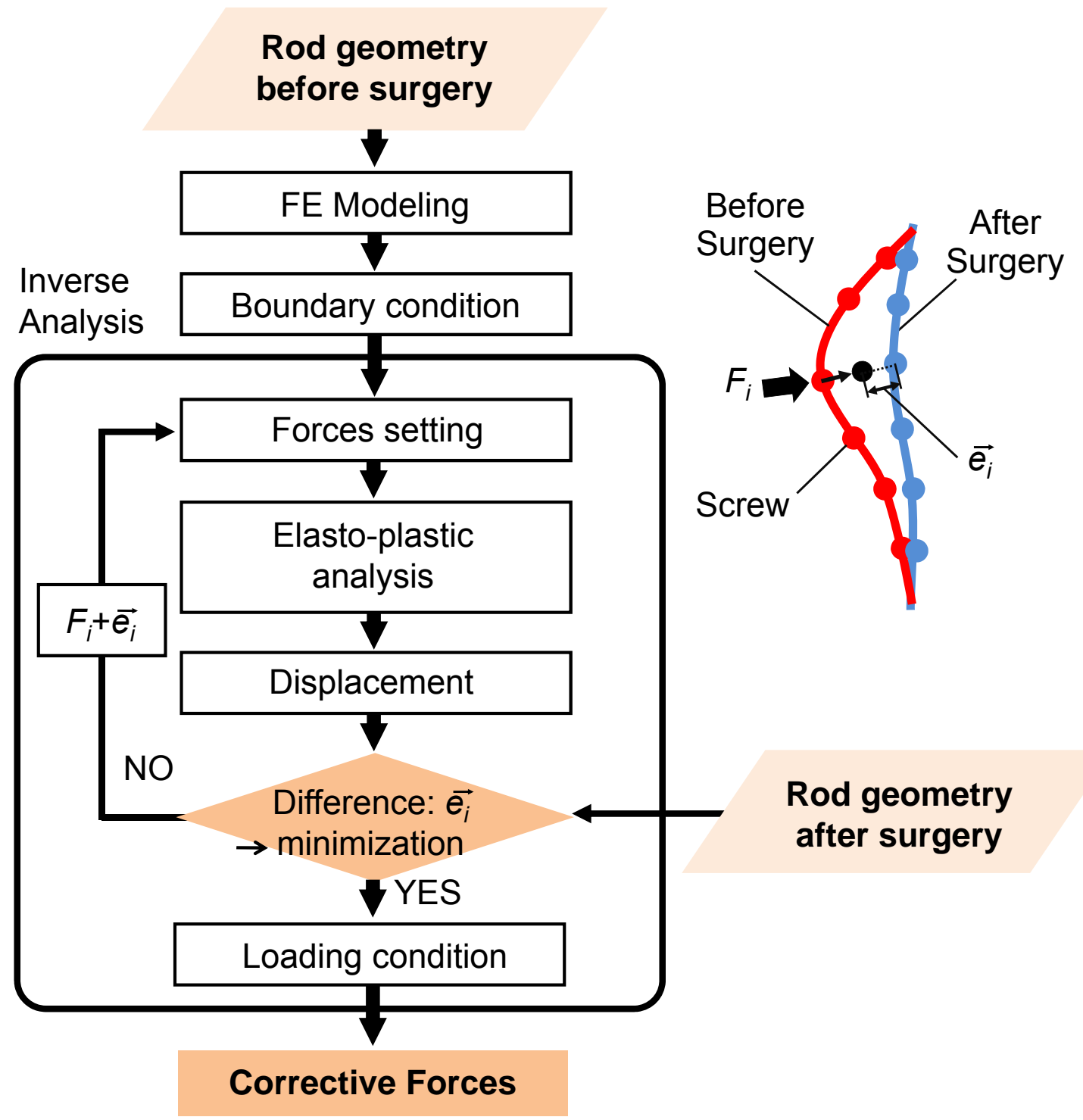

Figure 2 Procedure for corrective force analysis using FEA, redrawn (Salmingo et al., 2012a). 


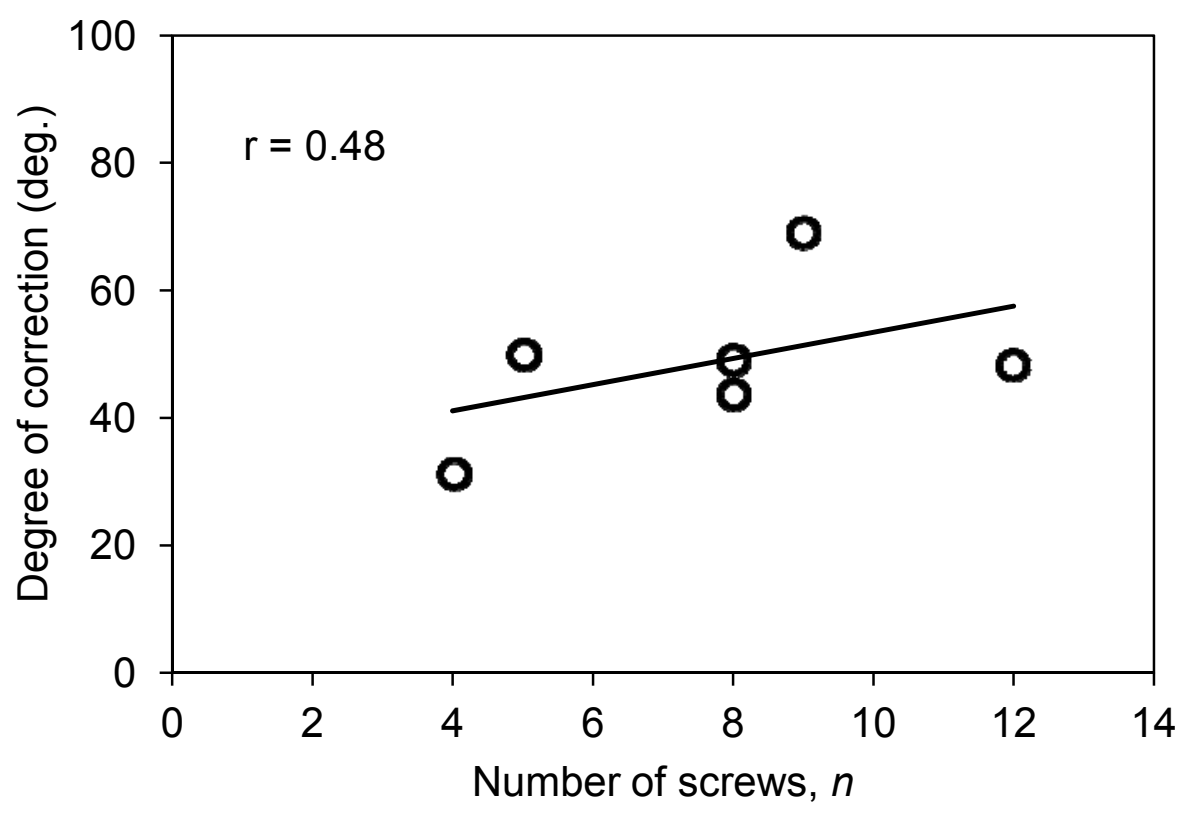

Figure 3 Relationship between the number of screws and degree of correction. 


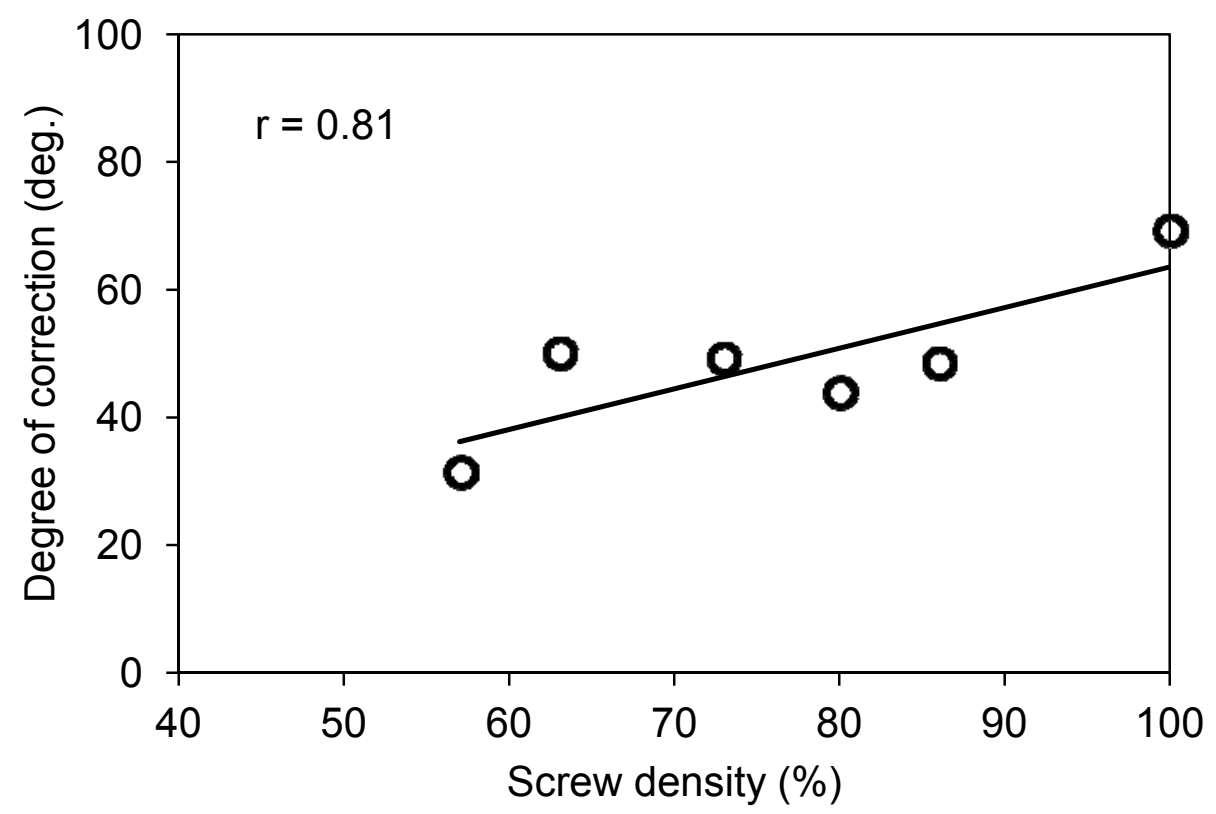

Figure 4 Relationship between screw density and degree of correction. 


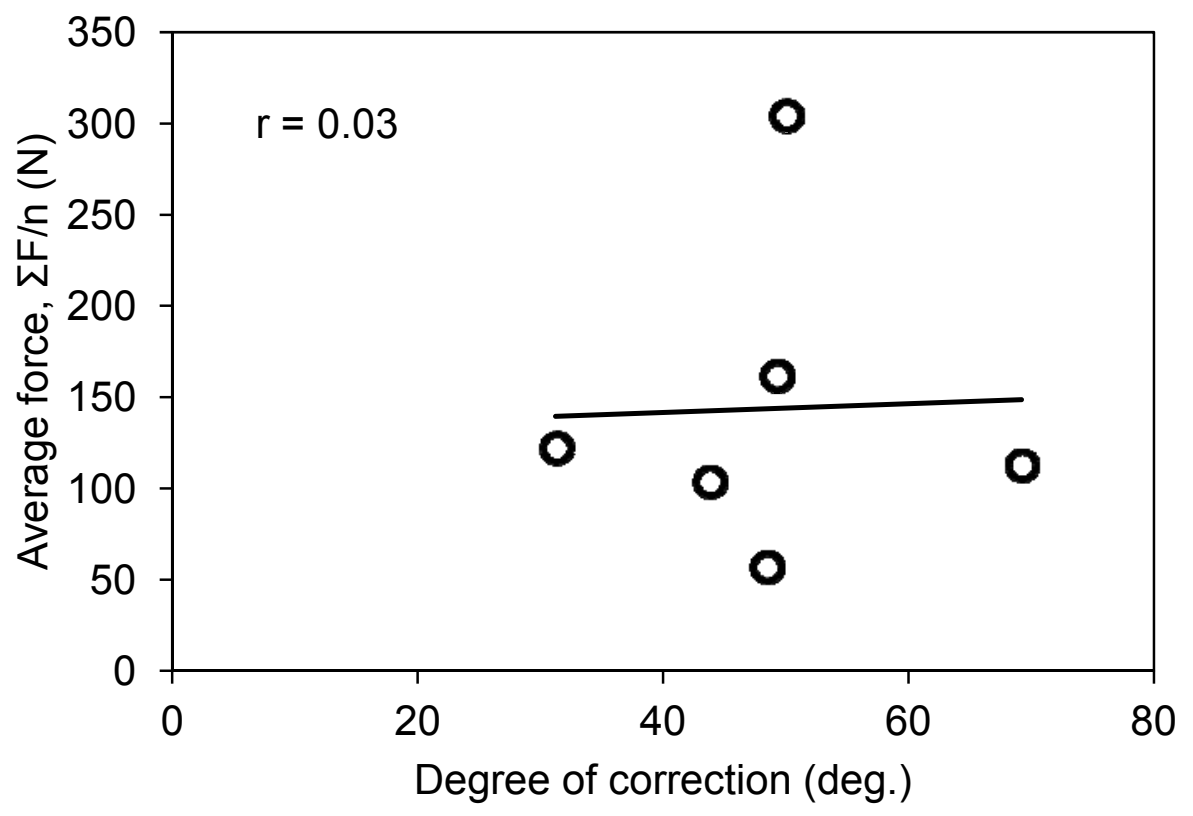

Figure 5 Relationship between degree of correction and average force. 

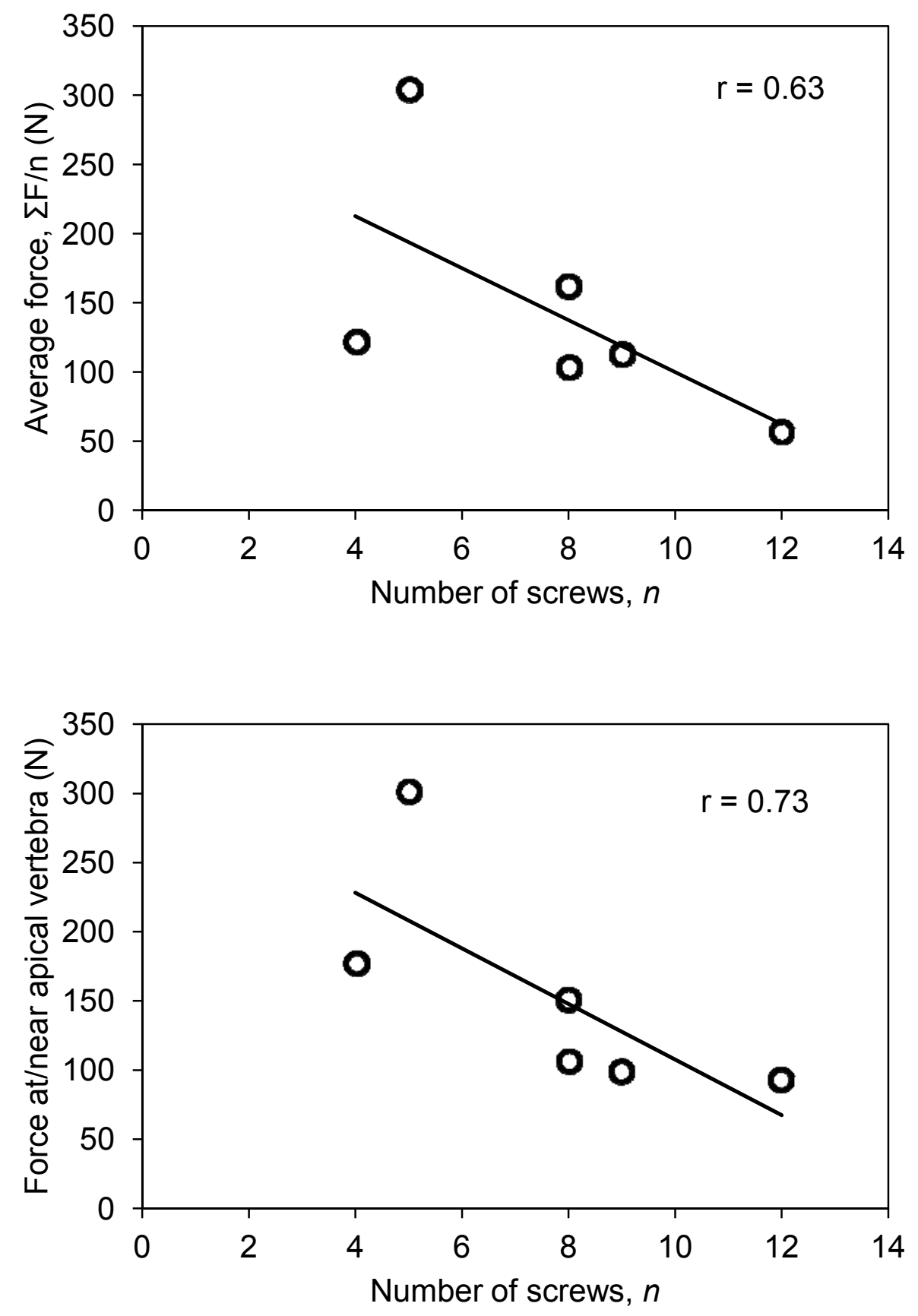

Figure 6 Relationships between number of screws and magnitude of forces. (a) Number of screws vs. average force (b) Number of screws vs. force at/near apical vertebra 

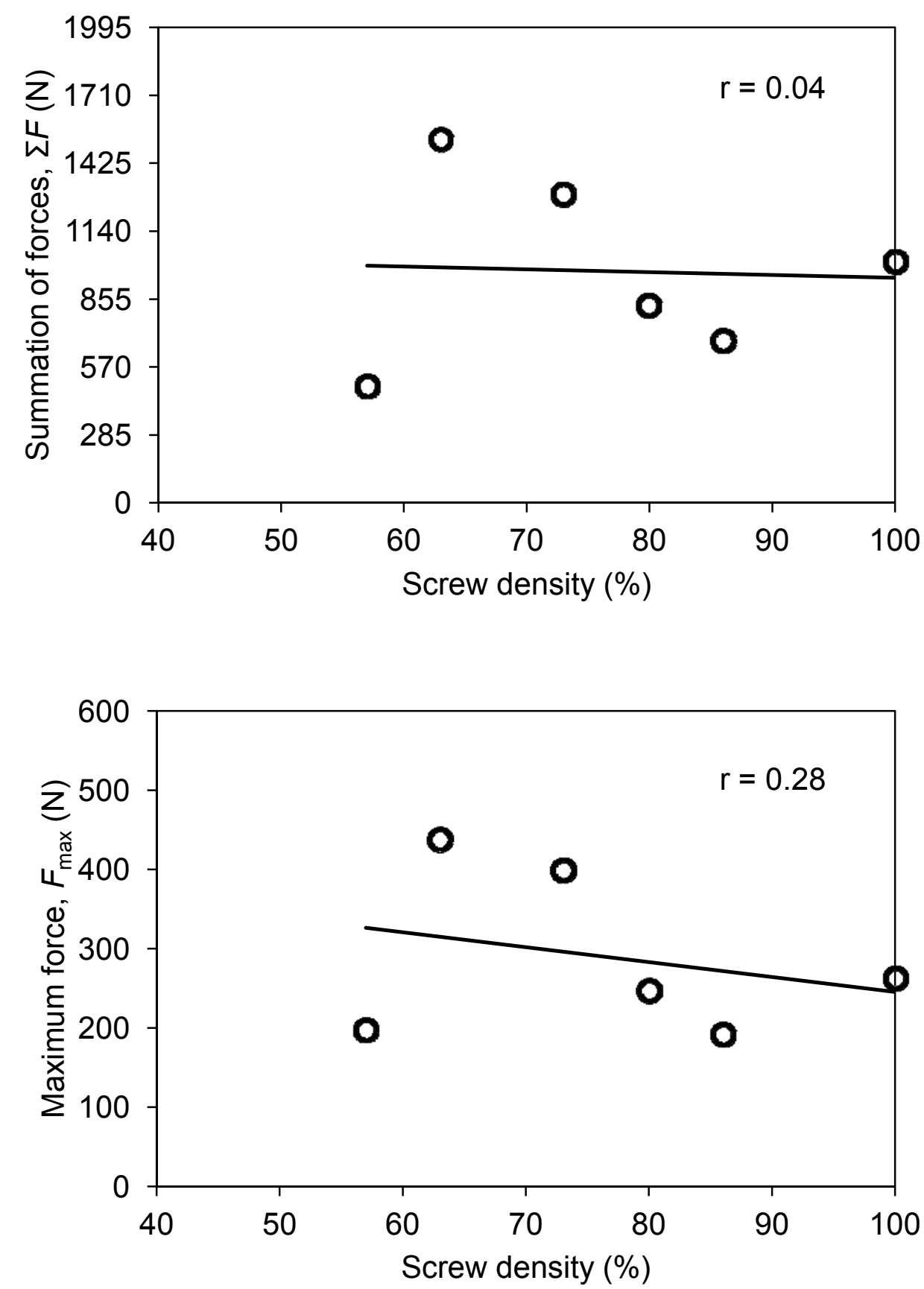

Figure 7 Relationships between screw density and magnitude of forces. (a) Screw density and summation of forces and (b) Screw density and maximum force 
Patient 1

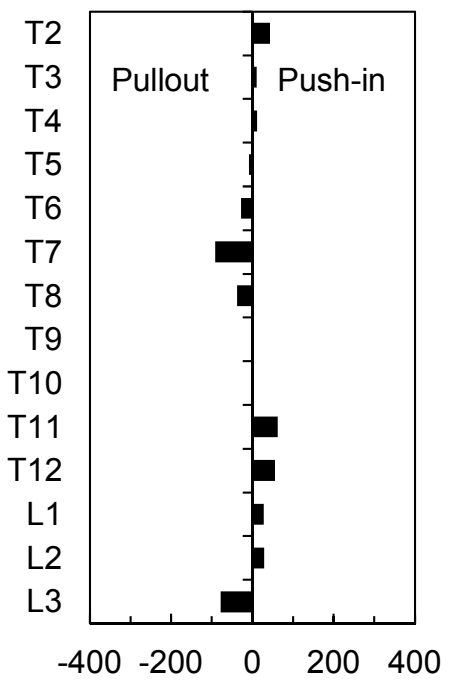

Patient 4

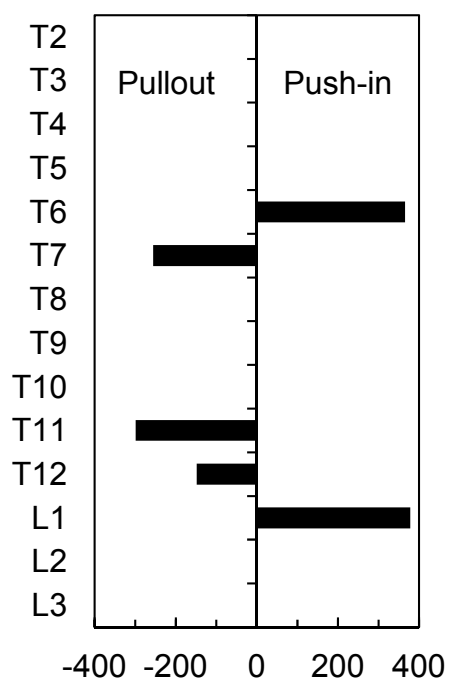

Patient 2

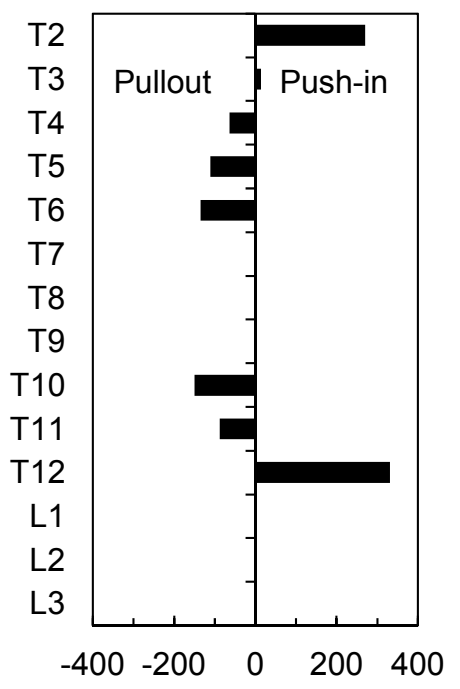

Patient 5

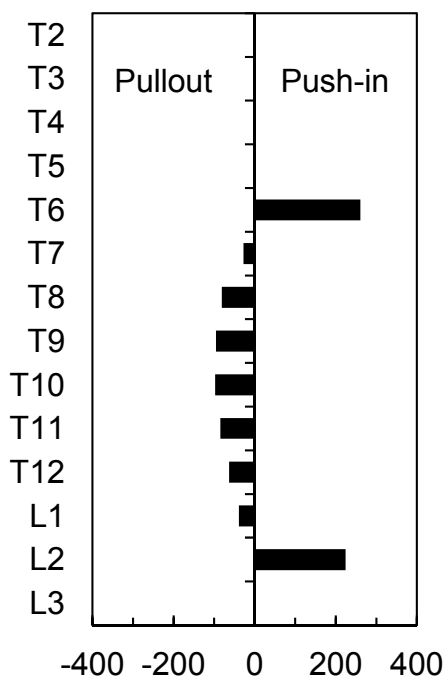

Patient 3

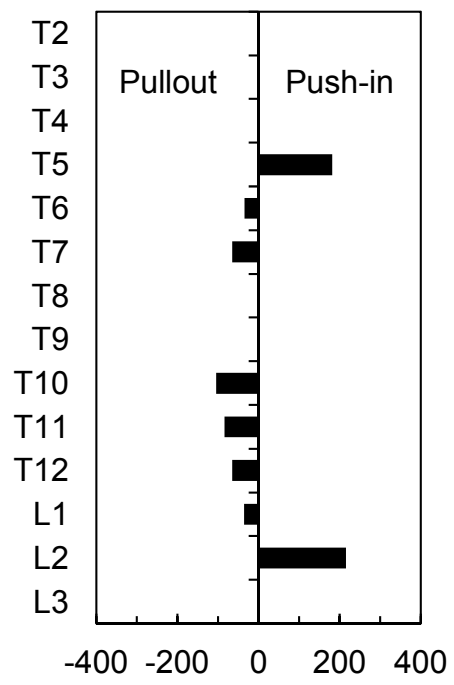

Patient 6

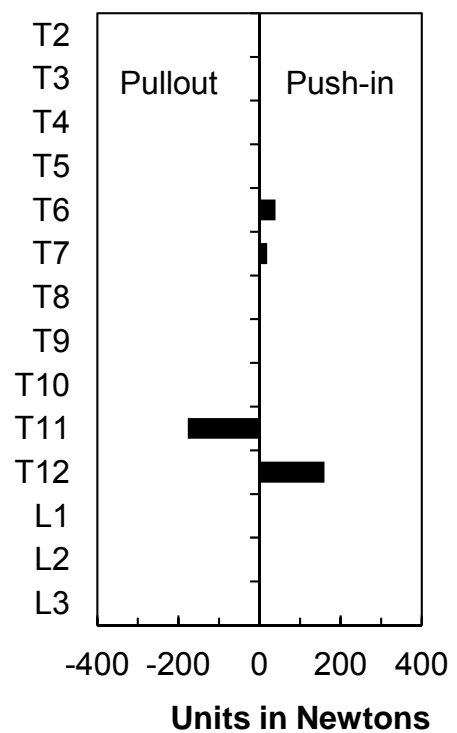

Figure 8 Pullout and push-in forces acting at the vertebra of each patient. 
Table 1 Clinical data of scoliosis patients.

\begin{tabular}{|c|c|c|c|c|c|c|}
\hline Patient & 1 & 2 & 3 & 4 & 5 & 6 \\
\hline $\begin{array}{c}\text { Preoperative Cobb angle, } \theta_{1} \\
\text { (deg.) }\end{array}$ & 76 & 75 & 57 & 68 & 83 & 59 \\
\hline $\begin{array}{c}\text { Postoperative Cobb angle, } \theta_{2} \\
\text { (deg.) }\end{array}$ & 27 & 26 & 13 & 18 & 14 & 28 \\
\hline $\begin{array}{c}\text { Degree of correction, } \Delta \theta=\theta_{1}-\theta_{2} \\
\text { (deg.) }\end{array}$ & 48 & 49 & 44 & 50 & 69 & 31 \\
\hline $\begin{array}{c}\text { Correction rate, } \Delta \theta / \theta_{1} \\
\text { (\%) }\end{array}$ & 64 & 65 & 77 & 73 & 83 & 53 \\
\hline
\end{tabular}


Table 2 Magnitude of corrective forces acting at each vertebra level.

\begin{tabular}{|c|c|c|c|c|c|c|}
\hline \multirow{2}{*}{$\begin{array}{c}\text { Vertebra } \\
\text { Level }\end{array}$} & \multicolumn{6}{|c|}{ Patient } \\
\hline & 1 & 2 & 3 & 4 & 5 & 6 \\
\hline T2 & 49 & 270 & & & & \\
\hline T3 & 33 & 58 & & & & \\
\hline T4 & 33 & 74 & & & & \\
\hline T5 & 31 & 115 & 182 & & & \\
\hline T6 & 36 & 139 & 43 & 375 & 263 & 59 \\
\hline T7 & - & - & 66 & 260 & 34 & 55 \\
\hline T8 & - & - & - & - & 82 & - \\
\hline T9 & ${ }^{*} 93$ & * & - & - & 96 & * \\
\hline$T 10$ & 39 & 151 & $*_{106}$ & * & ${ }^{*} 99$ & - \\
\hline$T 11$ & 63 & 89 & 84 & 301 & 85 & 177 \\
\hline T12 & 56 & 399 & 65 & 149 & 63 & 198 \\
\hline$L 1$ & 28 & & 35 & 439 & 39 & \\
\hline$L 2$ & 29 & & 248 & & 253 & \\
\hline L3 & 192 & & & & & \\
\hline
\end{tabular}


Table 3 Summary of number of screws, screw density and magnitude of forces of each patient.

\begin{tabular}{|c|r|r|r|r|r|r|}
\hline Patient & \multicolumn{1}{|c|}{1} & \multicolumn{1}{c|}{2} & \multicolumn{1}{c|}{3} & 4 & 5 & 6 \\
\hline Number of screws, $n$ & 12 & 8 & 8 & 5 & 9 & 4 \\
\hline Screw density (\%) & 86 & 73 & 80 & 63 & 100 & 57 \\
\hline Summation of forces, $\Sigma F(N)$ & 682 & 1295 & 829 & 1524 & 1014 & 489 \\
\hline Average force, $\Sigma F / n(N)$ & 57 & 162 & 104 & 305 & 113 & 122 \\
\hline Maximum force, $F_{\max }(N)$ & 192 & 399 & 248 & 439 & 263 & 198 \\
\hline Force at/near apex $(N)$ & 93 & 151 & 106 & 301 & 99 & 177 \\
\hline
\end{tabular}

